\title{
Forward and Diffractive Physics with Early ATLAS data
}

\author{
James Monk \\ On behalf of the ATLAS Collaboration ${ }^{1}$ \\ University College London, Gower St, London, WC1E 6BT, UK
}

\begin{abstract}
.
Proton-proton collisions at the Large Hadron Collider will soon commence and measurements of diffractive processes may be some of the first results produced. These proceedings outline some of the planned early measurements of diffractive physics and forward jets by the ATLAS collaboration.
\end{abstract}

Keywords: ATLAS, Diffraction, QCD

PACS: $12.38 . \mathrm{Qk}$

\section{INTRODUCTION}

Diffractive events at hadron colliders are typically identified by a region of the detector, known as a rapidity gap, that contains very little QCD radiation. In order to observe diffractive events in this way, the rapidity gap must not be filled in by radiation from either the underlying event or multiple proton-proton interactions. The latter becomes problematic once the instantaneous luminosity becomes large. The low luminosity period during the early $\mathrm{LHC}$ running is therefore the ideal time to collect diffractive data with the ATLAS detector.

\section{THE ATLAS CALORIMETER AND FORWARD DETECTORS}

The ATLAS hadronic calorimeter [1] consists of a barrel region covering the rapidity range $-1.7<\eta<1.7$ together with two end-cap calorimeters covering $1.7<|\eta|<3.2$. The calorimeter cell size is approximately $0.1 \times 0.1$ in $\eta-\phi$ in the central region, $|\eta|<2.5$, and $0.2 \times 0.2$ between 2.5 and 3.2. Forward of $|\eta|=3.2$ lies the forward calorimeter, which covers the region up to $|\eta|=4.9$.

Between the Barrel and end-cap lies the Minimum Bias Trigger Scintillator (MBTS), a scintillating disc covering $2.09<|\eta|<3.84$ that can be used for triggering. The LUCID detector [2] is a Cerenkov counter forward of the end-cap covering the range $5.6<|\eta|<5.9$, the main purpose of which is to act as a luminosity counter. However, LUCID may also be used for triggering.

\footnotetext{
${ }^{1}$ Presented at Diffraction 2008.
} 


\section{SIGNAL PROCESSES}

\section{Gaps Between Jets}

If two protons scatter off each other by exchanging a pomeron or other colour singlet then there are no sources of QCD radiation in the rapidity region between the outgoing protons or their remnants and activity is heavily suppressed in that region. An experimental signature for colour singlet exchange is thus a jet in each of the forward regions separated by a so-called "rapidity gap" in which there is greatly reduced radiation.

Colour singlet exchange has been observed by both the Tevatron [3, 4] and HERA $[5,6]$ experiments, and we aim to first rediscover this process before studying the dependence on the gap size and jet energies in the kinematic regime of the ATLAS detector. In particular, the fraction of events with suppressed activity in the gap is predicted by BFKL pomeron exchange to rise with both jet energy and the rapidity interval between the jets.

Colour singlet exchange also provides a useful early opportunity to study jet reconstruction and triggering in the forward calorimeter. In addition, colour singlet exchange has a very similar colour flow signature to vector boson fusion processes, which can only be studied after a substantial amount of data has been collected (at least $30 \mathrm{fb}^{-1}$ for Higgs boson production, for example). The experience gained from studying colour singlet exchange in early low luminosity running may help develop techniques for later vector boson fusion analyses.

The ATLAS detector can trigger on the presence of jets in the forward calorimeter using a trigger that requires an energy cluster of greater than $18 \mathrm{GeV}$ in both forward calorimeters. It is possible that an additional veto on the number of central tracks $(|\eta|<2.5)$ could be used to enrich the data sample with colour singlet exchange events.

\section{QCD Evolution in the Forward Region}

Events in which jets are separated by a large rapidity interval, $\Delta \eta$, may show a pattern of radiation that is quite different from that described by a DGLAP parton shower [7]. The BFKL equation describes parton splittings which, in contrast to DGLAP, are not strongly ordered in the transverse momentum, $K_{T}$, of the emitted parton. BFKL describes regions of phase space in which $\ln 1 / x$ is large $(x$ being the longitudinal momentum fraction of the proton held by a parton). BFKL evolution should allow events in which there are forward jets and central mini jets separated by $\Delta \eta \simeq 2$.

The ATLAS detector covers a rapidity range two units of rapidity larger than the Tevatron experiments. This, together with the larger collision energy, will allow QCD evolution to be studied over a completely new rapidity span, which will help to validate and tune models of parton showering at low $x$. As with colour singlet exchange, the experience of reconstructing and triggering jets in the forward calorimeter will be valuable. The triggering strategy will be almost identical to that for colour singlet exchange. 


\section{Single and Double Pomeron Exchange}

As long as its transverse momentum is sufficiently low, the emission of a colour singlet pomeron from a proton usually leaves the proton intact. A parton can be extracted from the pomeron to participate in a hard scatter. Measurements of pomeron exchange can probe diffractive parton distribution functions and can also determine the probability with which a rapidity gap survives after soft re-scattering effects. The signature for the exchange of a single pomeron (figure 1 left) is a rapidity gap in one forward region (the direction in which the intact proton went) and a pomeron remnant together with the hard scatter. In a double pomeron exchange (figure 1 right) a pomeron is emitted from both protons, so there is a rapidity gap on both sides and two pomeron remnants.

Di-jet production via both single and double pomeron exchange should be observable after a relatively small amount of data has been collected. The event rate for single pomeron exchange may be high enough that a dedicated trigger is not necessary. This is not the case for double pomeron exchange, and a dedicated trigger will improve the sample size for single pomeron exchange. A diffractive trigger will have to veto activity in one or both forward regions for single or double pomeron exchange, respectively. The inner wheel of the ATLAS MBTS covers a rapidity range $2.82<\eta<3.84$, so triggering events without activity in the inner MBTS, together with a central energy cluster above $18 \mathrm{GeV}$ could provide a sample of single or double diffractive events. LUCID could additionally be used to veto activity in the forward region right up to $|\eta|<5$.9.
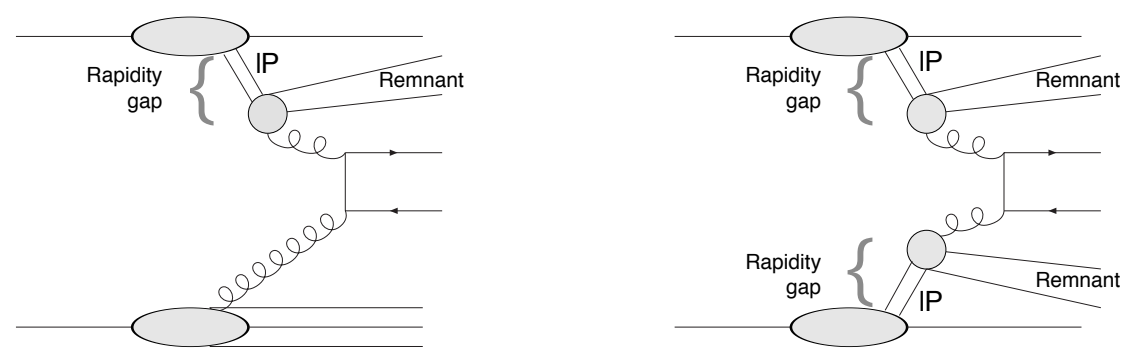

FIGURE 1. Single (left) and double (right) pomeron exchange.

\section{Central Exclusive Production}

Like double pomeron exchange, central exclusive production (CEP, figure 2) leaves the outgoing protons intact. Unlike double pomeron exchange, there are no pomeron remnants. Central exclusive di-jet production therefore produces a pair of central jets and nothing else. Another feature of CEP is that at leading order the central system must have even parity and zero spin [8]. If the outgoing protons are measured downstream of the main ATLAS detector, as has been proposed [9], then the mass and spin of the central system can be measured with high accuracy. In the case that the central system is a Higgs boson candidate, for example, this technique could provide both a precise measurement of the Higgs mass and verification that the observed resonance is spin zero. 
Observation of central exclusive di-jets by the CDF collaboration [10] is in agreement with theoretical expectations. However, the cross section for central exclusive production has a large theoretical uncertainty and an early measurement of exclusive di-jet production at the LHC energy will be enormously useful [11] as a step towards a possible later central exclusive resonance search using forward proton taggers. The required proton taggers at a distance of 420 metres away from ATLAS will not be installed during early running. However, the presence of central exclusive di-jets can be inferred with the main ATLAS detector using the distribution of the quantity $R_{j j} . R_{j j}$ is defined as

$$
R_{j j}=\frac{M_{j j}}{\sqrt{s_{c a l}}}
$$

where $M_{j j}$ is the invariant mass of the di-jet system and $\sqrt{s_{c a l}}$ is the total invariant mass deposited in the detector. For a completely exclusive event in the absence of hadronisation and detector effects, $R_{j j}=1$.

Data for central exclusive di-jets could be captured using a similar $18 \mathrm{GeV}$ cluster plus MBTS veto trigger combination to double pomeron exchange. Double pomeron exchange is in fact a background to central exclusive di-jets and one of the key contributions to the theoretical uncertainty in the cross sections for both processes is the effect that soft underlying physics has on the gap survival. The relative rates of non-diffractive, single and double pomeron exchange and central exclusive di-jets should provide useful insight to the soft survival probability.

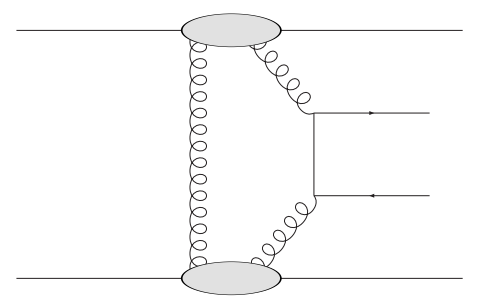

FIGURE 2. Central Exclusive Production of a pair of quarks

\section{REFERENCES}

1. ATLAS collaboration, CERN-LHCC-96-040

2. ATLAS collaboration, CERN-LHCC-2004-010

3. B. Abbott et al. [D0 Collaboration], Phys. Lett. B 440 (1998) 189 [arXiv:hep-ex/9809016].

4. F. Abe et al. [CDF Collaboration], Phys. Rev. Lett. 80 (1998) 1156.

5. M. Derrick et al. [ZEUS Collaboration], Phys. Lett. B 369, 55 (1996) [arXiv:hep-ex/9510012].

6. C. Adloff et al. [H1 Collaboration], Eur. Phys. J. C 24, 517 (2002) [arXiv:hep-ex/0203011].

7. A. H. Mueller and H. Navelet, Nucl. Phys. B 282 (1987) 727.

8. V. A. Khoze, A. D. Martin and M. G. Ryskin, Eur. Phys. J. C 19 (2001) 477 [Erratum-ibid. C 20 (2001) 599] [arXiv:hep-ph/0011393].

9. M. G. Albrow et al. [FP420 R\&D Collaboration], FERMILAB-FN-0825-E [arXiv:0806.0302 [hepex]].

10. T. Aaltonen et al. [CDF Collaboration], Phys. Rev. D 77, 052004 (2008) [arXiv:0712.0604 [hep-ex]].

11. V. A. Khoze, A. D. Martin and M. G. Ryskin, Eur. Phys. J. C 55 (2008) 363 [arXiv:0802.0177 [hep$\mathrm{ph}]$. 